lignen Neoplasien in den bestrahlten $\mathrm{Re}$ gionen auf. Schilddrüsenkarzinome wurden nur bei Patienten beobachtet, die im Hals- oder Mediastinalbereich bestrahlt worden waren.

Die Autoren schlussfolgern, dass $\mathrm{Pa}$ tienten, die wegen eines Hodgkin-Lymphoms im Kindes- und Jugendalter bestrahlt worden waren, zur Früherkennung therapiebedingter Spätfolgen insbesondere von sekundären malignen Neoplasien - lebenslang eine regelmäßige ärztliche Nachsorge erfahren sollen.

Dörffel W et al. Secondary malignancies following treatment for hodgkin's lymphoma in childhood and adolescence. Dtsch Arztebl Int 2015;112:320-7

\section{Kommentar}

Die Ergebnisse zeigen eindrücklich, dass Spätfolgen, insbesondere sekundäre maligne Neoplasien, langfristig die exzellente Prognose hinsichtlich des Überlebens bei Kindern und Jugendlichen mit HodgkinLymphom beeinträchtigen können. Neben der regelmäßigen ärztlichen Nachsorge versucht man deshalb derzeit, die Strahlentherapie bei Kindern und Jugendlichen weiter zu reduzieren oder gar zu vermeiden. Hierbei ist es wichtig, eine möglicherweise erhöhte Inzidenz von Rezidiven bei Verzicht auf eine Bestrahlung gegenüber der erhöhten Inzidenz von sekundären malignen Neoplasien bei Bestrahlung abzuwägen. Dies kann aber nur durch eine genaue Nachbeobachtung über viele Jahre erreicht werden. Prof.Dr.Thomas Lehrnbecher

\title{
Budesonid vermindert chronische Lungenerkrankung
}

Pränatale systemische Glukokortikoide vermindern das Risiko einer bronchopulmonalen Dysplasie (BPD) bei extremen Frühgeborenen. Nun wurde in einer Studie die Wirkung von inhalativen Glukokortikoiden abgeklärt.

n einer randomisierten Multicenterstudie an der Universitätskinderklinik in Tübingen wurden 863 beatmungsbedürftige Frühgeborene mit einem Gestationsalter zwischen $23+0$ und $27+6$ SSW untersucht. Unterschieden wurde zwischen einer Interventionsgruppe, bei der 24 Stunden nach der Geburt eine inhalative Budesonidbehandlung begonnen und fortgesetzt wurde bis kein zusätzlicher Sauerstoff und keine respiratorische Unterstützung mit positivem Atemdruck mehr erforderlich oder ein Gestationsalter von $32+0$ SSW erreicht waren, und einer Placebogruppe.

Die Inzidenz der BPD in der Budesonid- beziehungsweise der Placebogruppe betrug 27,8 beziehungsweise $38 \%$ (p $=0,004$ ), allerdings war die Mortalität in der Budesonidgruppe mit einem $\mathrm{p}=$ 0,17 etwas erhöht. Erfreulicherweise waren die Anzahl von operativ anzugehenden PDAs und die Zahl der Reintubationen in der Budesonid-Gruppe signifikant geringer.
Bassler D et al. Early inhaled budesonide for the prevention of bronchopulmonary dysplasia. N Engl J Med 2015;373:1497-506

\section{Kommentar}

Die Ergebnisse zeigen, dass inhalatives Budesonid bezüglich einer BPD-Entwicklung von extrem unreifen Frühgeborenen bei frühzeitigem Einsatz einen signifikant positiven Effekt im Vergleich zu Placebo hat. Überraschend und unerklärt zeigte sich in dieser Untersuchung an 40 europäischen Zentren jedoch eine erhöhte Mortalität in der Budesonid-Gruppe. Die Auswertung des entwicklungsneurologischen Outcomes erwarten wir mit Spannung, zumal von systemisch applizierten Glukokortikoiden belegt ist, dass sie nachweislich bezüglich der späteren Hirnentwicklung von Frühgeborenen schlecht abschneiden. Einmal mehr ist das Gesamtkonzept des Teams gefragt - bis hin zu Methoden wie "less invasive surfactant administration (LISA)" zur BPD-Vermeidung und effektiven Qualitätsentwicklung in der Neonatologie. Dr. Thomas Hoppen 